\title{
An equation of state for liquid iron and implications for the Earth's core
}

\author{
William W. Anderson and Thomas J. Ahrens \\ Lindhurst Laboratory of Experimental Geophysics, Seismological Laboratory, California Institute of \\ Technology, Pasadena
}

\begin{abstract}
An equation of state is presented for liquid iron based on published ultrasonic, thermal expansion, and enthalpy data at 1 bar and on pulse-heating and shock wave compression and sound speed data up to $10 \mathrm{Mbar}$. The equation of state parameters, centered at $1 \mathrm{bar}$ and $1811 \mathrm{~K}$ (the normal melting point of iron), are density, $\rho_{0}=7019 \mathrm{~kg} / \mathrm{m}^{3}$, isentropic bulk modulus, $K_{S 0}=109.7 \mathrm{GPa}$, and the firstand second-pressure derivatives of $K_{S}, K_{s 0}^{\prime}=4.66$ and $K_{s 0}^{\prime \prime}=-0.043 \mathrm{GPa}^{-1}$. A parameterization of the Grüneisen parameter $\gamma$ as a function of density $\rho$ and specific internal energy $E$ is $\gamma=\gamma_{0}+\gamma^{\prime}\left(\rho / \rho_{0}\right)^{n}\left(E-E_{0}\right)$ where $\gamma_{0}=1.735, \gamma^{\prime}=-0.130$ $\mathrm{kg} / \mathrm{MJ}, n=-1.87$, and $E_{0}$ is the internal energy of the liquid at $1 \mathrm{bar}$ and $1811 \mathrm{~K}$. The model gives the temperature dependence of $\gamma$ at constant volume as $\left.(\partial \gamma / \partial T)_{V}\right|_{1 \text { bar, } 1811 \mathrm{~K}}=-8.4 \times 10^{-5} \mathrm{~K}^{-1}$. The constant volume specific heat of liquid $\mathrm{Fe}$ at core conditions is 4.0-4.5 R. The model gives excellent agreement with measured temperatures of $\mathrm{Fe}$ under shock compression. Comparison with a preliminary reference Earth model indicates that the light component of the core does not significantly affect the magnitude of the isentropic bulk modulus of liquid $\mathrm{Fe}$ but does decrease its pressure derivative by $\sim 10 \%$. Pure liquid $\mathrm{Fe}$ is $3-6 \%$ more dense than the inner core, supporting the presence of several percent of light elements in the inner core.
\end{abstract}

\section{Introduction}

Liquid iron, which in impure form makes up the outer core, is one of the most abundant and important phases in Earth's interior. An accurate description of its properties at high pressure is therefore of great interest for theoretical studies of Earth's interior. In this study, experimental data are used to constrain the thermodynamic behavior of liquid $\mathrm{Fe}$ and to compare its properties with those of Earth's core.

Several previous studies have considered the equation of state of liquid iron but were limited by the available data. Jeanloz [1979] used the then available shock wave data for porous and nonporous samples to develop a general pressure-volume-temperature equation of state (EOS) for both liquid and solid iron. Stevenson [1980, 1981], employing liquid state theory to develop a simple model, demonstrated the general trends of properties for liquid Fe at high pressures. Anderson [1986] presented equations of state for the various phases of $\mathrm{Fe}$ based on theory and experimental data, concentrating primarily on the solid phases.

More recently, Svendsen et al. [1989] presented an equation of state for liquid iron, constraining parameters for a Yukawa pair potential by assuming that liquid structure is constant along the liquidus and applying their analysis to the melting curve of Fe from Williams et al. [1987] to determine the values of adjustable EOS parameters. Most recently, Grover [1990] presented a theoretical two-phase solid-liquid EOS with parameters constrained by theory and by fitting shock wave data from porous and nonporous samples.

Copyright 1994 by the American Geophysical Union.

Paper number 93JB03158.

0148-0227/94/93JB-03158\$05.00

\section{Constraints on the Equation of State Reference Condition and Properties}

Traditionally, equations of state used in geophysics are referenced to normal ambient conditions ( 1 bar, $298 \mathrm{~K}$ ). We choose instead to use the normal melting point of pure $\mathrm{Fe}(P$ $=1 \mathrm{bar}, T_{m}=1811 \mathrm{~K}$ ) for the present model, because the properties of the liquid phase at the melting point can be measured directly, whereas the properties at $298 \mathrm{~K}$ cannot. Hence for this discussion the reference pressure and temperature are $P_{0}=1$ bar $(0.1 \mathrm{MPa})$ and $T_{0}=1811 \mathrm{~K}$.

Density and thermal expansion coefficient. Numerous experimental studies have examined the density of liquid $\mathrm{Fe}$ at 1 bar [Drotning, 1981; Basin et al., 1979; Lucas, 1972; Ivakhnenko and Kashin, 1976]. They find densities at the melting point $\rho_{0}$ ranging from 7015 to $7150 \mathrm{~kg} / \mathrm{m}^{3}$, with most recent results falling in the range from 7015 to $7070 \mathrm{~kg} / \mathrm{m}^{3}$. Drotning [1981] studied the density and thermal expansion to above $2300 \mathrm{~K}$ and presented an assessment of sources of error. Analyses by Drotning [1981] and Basin et al. [1979] suggest that the most reliable data are those of Drotning [1981] and Lucas [1972] (Figure 1). We fit these data to

$$
\begin{aligned}
V\left(\mathrm{~m}^{3} / \mathrm{kg}\right)= & 1 / \rho=1.4247( \pm 0.0004) \times 10^{-4} \\
& +1.3105( \pm 0.0195) \times 10^{-8}\left(T-T_{0}\right)
\end{aligned}
$$

This fit gives $\rho_{0}=7019 \pm 2 \mathrm{~kg} / \mathrm{m}^{3}$ and $\left.(\partial \rho / \partial T)_{P}\right|_{1811 \mathrm{~K}}=$ $-0.646 \pm 0.010 \mathrm{~kg} \mathrm{~m}^{-3} \mathrm{~K}^{-1}$. The corresponding value of the volume thermal expansion coefficient is $\alpha=(\partial \ln V / \partial T)_{P}=$ $9.20( \pm 0.14) \times 10^{-5} \mathrm{~K}^{-1}$ at the reference point.

We note here the pulse-heating experiments of Hixson et al. [1990]. They measure $\rho$ at $P=0.2 \mathrm{GPa}$ over the temperature range from $2125 \mathrm{~K}$ to $3950 \mathrm{~K}$, obtaining $\rho_{0}=$ $6994 \mathrm{~kg} / \mathrm{m}^{3}$ and $(\partial \rho / \partial T)=-0.64985 \mathrm{~kg} \mathrm{~m}^{-3} \mathrm{~K}^{-1}$ with a 


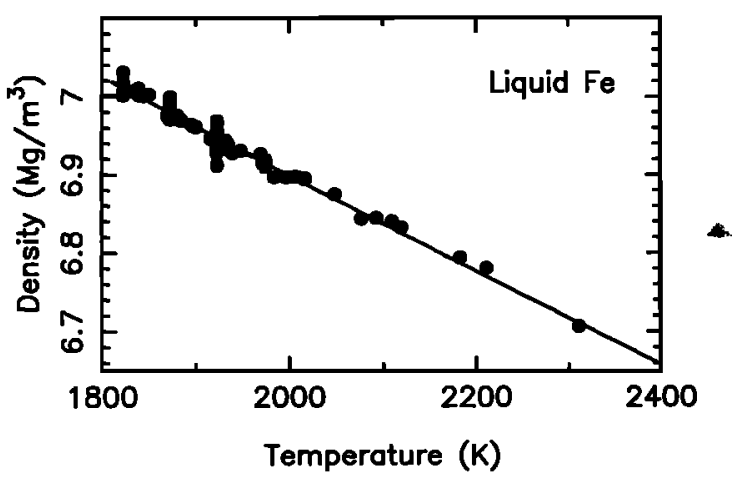

Figure 1. Density data of Lucas [1972] and Drotning [1981] for liquid $\mathrm{Fe}$ at $1 \mathrm{bar}$, with the best fit curve assuming that volume varies linearly with $T$.

linear fit of $\rho$ versus $T$. Within the stated uncertainties, these data are consistent with the adopted expression for $V$.

Reference heat capacity and transition energy. The thermodynamic functions of Fe have been extensively studied at atmospheric pressure [e.g., Desai, 1986]. We choose Desai's [1986] preferred value of the specific heat: $C_{P}=835 \pm 54$ $\mathrm{J} \mathrm{kg}^{-1} \mathrm{~K}^{-1}$ at 1 bar and $1811 \mathrm{~K}$. The pulse-heating data of Hixson et al. [1990] for enthalpy $H$ versus $T$ give $C_{P}=$ $815.4 \mathrm{~J} \mathrm{~kg}^{-1} \mathrm{~K}^{-1}$, in good agreement with Desai [1986].

We also want the internal energy $E_{t r}$ required to transform Fe from the $\alpha$ phase at 1 bar and $298 \mathrm{~K}$ to the liquid at $P_{0}$ and $T_{0}$

$$
E_{t r}=E_{\mathrm{liq}, 1 \mathrm{bar}, 1811 \mathrm{~K}}-E_{\alpha, 1 \mathrm{bar}, 298 \mathrm{~K}}
$$

Desai [1986] presents preferred values of enthalpy changes across phase transitions and the heat capacity as a function of temperature in the solid, so that the value of the enthalpy difference $\Delta H_{t r}$ between the liquid $F e$ at the reference point and $\alpha$-Fe at 1 bar and $298 \mathrm{~K}$ can be obtained from

$$
\Delta H_{t r}=\int_{298 \mathrm{~K}}^{T_{0}} C_{P} d T+\Delta H_{\alpha \rightarrow \gamma}+\Delta H_{\gamma \rightarrow \delta}+\Delta H_{\delta \rightarrow \text { liq }}
$$

From the relation between enthalpy and internal energy, we have

$$
E_{t r}=\Delta H_{t r}+P \Delta V
$$

At 1 bar, $P \Delta V$ is negligible, so $E_{t r}=\Delta H_{t r}$. Thus from the values of $C_{p}$ and $\Delta H$ from Desai [1986], $E_{t r}=1.3007$ ( \pm $0.0118) \times 10^{6} \mathrm{~J} / \mathrm{kg}$.

Bulk modulus. Although we can use shock wave equation of state (SWEOS) data to get the reference point isentropic bulk modulus $K_{S 0}=-\left.V(\partial V / \partial T)_{S}\right|_{P o, T o}$, measurements of the bulk sound speed $V_{b}$ give $K_{s 0}$ more directly and with greater accuracy from

$$
V_{b}=\left(K_{S} / \rho\right)^{1 / 2}
$$

Kéita et al. [1982] obtain $V_{b}=3912 \mathrm{~m} / \mathrm{s}$ and $\left(\partial V_{b} / \partial T\right)_{P}=$ $-0.22 \mathrm{~m} \mathrm{~s}^{-1} \mathrm{~K}^{-1}$ at the melting point, whereas Casas et al. [1984] obtain $V_{b}=4052 \pm 134 \mathrm{~m} / \mathrm{s}$ at the melting point. Fillipov et al. [1966] find an anomalously high value of $V_{b}=$ $4400 \mathrm{~m} / \mathrm{s}$. We combine the data of Kurz and Lux [1969] and Tsu et al. [1985] to cover a range of $\sim 140 \mathrm{~K}$. These data are shown in Figure 2 together with a straight-line fit (similar to previous studies) of $V_{b 0}=3954 \pm 24 \mathrm{~m} / \mathrm{s}$ and $\left(\partial V_{b} / \partial T\right)_{P}=$ $-0.54 \pm 0.21 \mathrm{~m} \mathrm{~s}^{-1} \mathrm{~K}^{-1}$ at the reference point. Shaner et al. [1988] suggest that $V_{b}$ would be better represented as a linear function of $\rho$ for most liquid metals. However, the density variation over this temperature range is so close to linear that a linear fit in $T$ gives the same results. With (5) and $\rho_{0}$, we get $K_{S 0}=109.7 \pm 0.7 \mathrm{GPa}$ at 1 bar and $1811 \mathrm{~K}$ which is also consistent with the data of Hixson et al. [1990].

\section{High Pressure Quantities}

Shock Hugoniot of iron. The shock Hugoniot is described by a polynomial giving the velocity of the shock wave $U_{s}$ as a function of the particle velocity of the shocked material $u_{p}$ in the rest frame of the unshocked material

$$
U_{s}=C_{0}+s u_{p}+s^{\prime} u_{p}^{2}+\cdots
$$

The shock state pressure $\boldsymbol{P}_{H}$, density $\rho_{H}$, and specific internal energy $E_{H}$ are obtained via the Rankine-Hugoniot equations for conservation of mass, momentum, and energy

$$
\begin{gathered}
P_{H}-P_{0}=\rho_{00} U_{s} u_{p} \\
\rho_{H} / \rho_{00}=U_{s} /\left(U_{s}-u_{p}\right) \\
E_{H}-E_{0}=\frac{1}{2}\left(P_{H}+P_{0}\right)\left(V_{00}-V_{H}\right)=\frac{1}{2} u_{p}^{2}
\end{gathered}
$$

where the subscript 00 refers to the sample before being shocked. The principal Hugoniot curve describes shocks from the equilibrium state at $P_{0}=1$ bar and $T=298 \mathrm{~K}$ in a sample having no porosity.

Three sets of parameters for the principal Hugoniot curve of Fe from McQueen et al. [1970], Al'tshuler et al. [1981], and Brown and McQueen [1986] have found widespread use in the literature. However, these parameters are based on data in the stability field of solid Fe. To avoid possible systematic errors arising from use of Hugoniot curves constrained by solid state data, we fit (6) to the available data from that portion of the principal Hugoniot of Fe that falls in the liquid stability region, as delineated by the sound speed data of Brown and McQueen [1986]. The data are listed in Table 1, although we exclude the highest pressure point $(1351 \mathrm{GPa})$ from the fit because it seems to fall away from the trend established by the other data. In cases where authors do not give uncertainties for their data, we use the analysis of Al'tshuler et al. [1981] to estimate the uncertainties. The resulting fit (Figure 3) gives $C_{0}=3861 \pm 839 \mathrm{~m} / \mathrm{s}, s=$

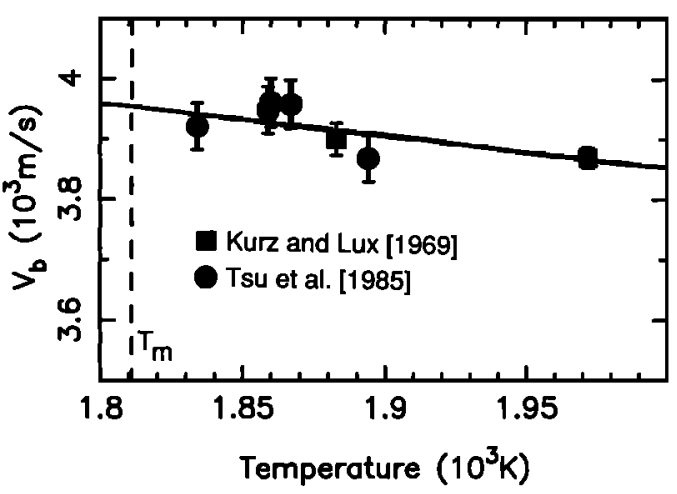

Figure 2. Sound speed data for liquid Fe at 1 bar, with a linear fit as a function of $T$. 
Table 1. Data for the Liquid Phase Portion of the Iron Principal Hugoniot

\begin{tabular}{|c|c|c|c|c|c|}
\hline$\rho_{0}, \mathrm{~kg} / \mathrm{m}^{3}$ & $U_{s}, \mathrm{~m} / \mathrm{s}$ & $u_{p}, \mathrm{~m} / \mathrm{s}$ & $\rho_{H}, \mathrm{~kg} / \mathrm{m}^{3}$ & $P_{H}, \mathrm{GPa}$ & \\
\hline 7851 & $10200 \pm 102$ & $4050 \pm 41$ & $13021 \pm 177$ & $324.3 \pm 6.1$ & Marsh [1980] \\
\hline 7851 & $10350 \pm 104$ & $4070 \pm 41$ & $12939 \pm 173$ & $330.7 \pm 6.2$ & Marsh [1980] \\
\hline 7850 & $17740 \pm 176$ & $9700 \pm 24$ & $17317 \pm 566$ & $1351 \pm 97$ & Al'tshuler et al. [1981] \\
\hline 7850 & $9980 \pm 100$ & $3830 \pm 24$ & $12740 \pm 112$ & $300 \pm 6.2$ & Al'tshuler et al. [1958] \\
\hline 7850 & $10450 \pm 105$ & $4200 \pm 42$ & $13130 \pm 125$ & $344 \pm 7.2$ & Al'tshuler et al. [1958] \\
\hline 7850 & $10670 \pm 107$ & $4320 \pm 43$ & $13190 \pm 127$ & $362 \pm 7.5$ & Al'tshuler et al. [1958] \\
\hline 7850 & $11100 \pm 111$ & $4590 \pm 46$ & $13380 \pm 133$ & $400 \pm 8.3$ & Al'tshuler et al. [1958] \\
\hline 7850 & $11320 \pm 113$ & $4830 \pm 48$ & $13690 \pm 144$ & $429 \pm 8.9$ & Al'tshuler et al. [1958] \\
\hline 7850 & $12000 \pm 120$ & $5170 \pm 52$ & $13790 \pm 148$ & $487 \pm 10.1$ & Al'tshuler et al. [1958] \\
\hline 7850 & $15500 \pm 155$ & $7710 \pm 77$ & $15622 \pm 547$ & $938 \pm 33$ & Krupnikov et al. [1963] \\
\hline 7850 & $15150 \pm 151$ & $7520 \pm 75$ & $15582 \pm 545$ & $894 \pm 32$ & Krupnikov et al. [1963] \\
\hline 7850 & $15100 \pm 151$ & $7340 \pm 73$ & $15284 \pm 535$ & $870 \pm 32$ & Al'tshuler et al. [1962] \\
\hline
\end{tabular}

$1.656 \pm 0.309$, and $s^{\prime}=-1.945( \pm 2.66) \times 10^{-5} \mathrm{~s} / \mathrm{m}$. The fit from Al'tshuler et al. [1981] is similar to the present result but has a much greater slope at the low $u_{p}$ end than is justified by the liquid state data. It should be noted that the quadratic term is not statistically significant with the present data set, but results presented later in this discussion justify retaining the quadratic term.

Grüneisen parameter. The thermodynamic Grüneisen parameter $\gamma$ is defined by

$$
\gamma=V(\partial P / \partial E)_{V}
$$

Recently, new data for liquid Fe have become available which allow $\gamma$ to be studied in greater detail than before. Let us begin by examining the available data for $\gamma$.

Data for $\gamma$ at $P_{0}$ and $T_{0}$. The value of $\gamma$ at the reference point is obtained from other properties via

$$
\gamma=\frac{\alpha K_{S}}{\rho C_{P}}=\frac{\alpha K_{T}}{\rho C_{V}}
$$

Using the values of $\rho_{0}, \alpha_{0}, K_{S 0}$, and $C_{P 0}$, we get $\gamma_{0}=1.723$ \pm 0.116 . We will use this value as a datum but only require the final fit to fall within its limits of uncertainty.

Pulse-heating experiments. Recently, Hixson et al. [1990]
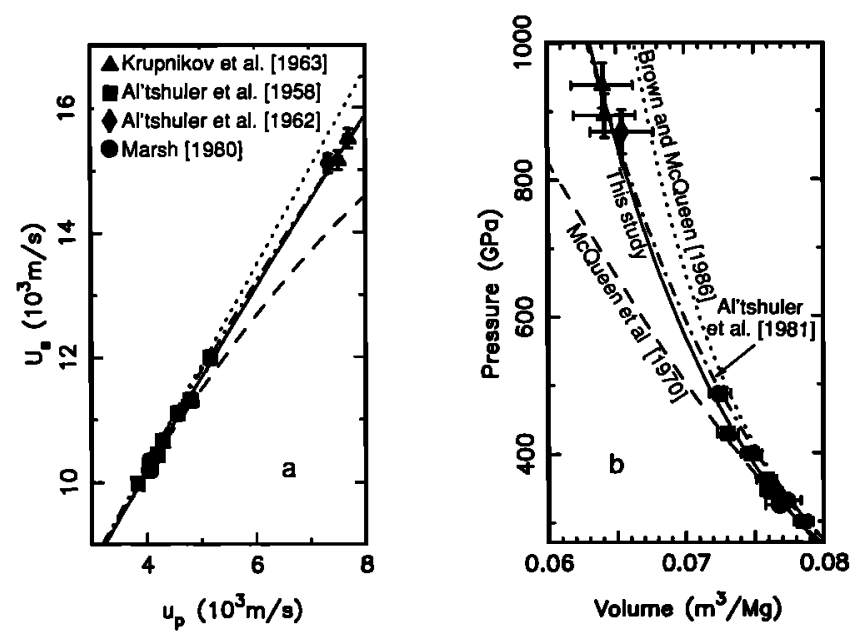

Figure 3. Principal Hugoniots for nonporous Fe compared with data in the liquid $\mathrm{Fe}$ stability region. (a) $U_{s}-u_{p}$ projection. (b) $P-V$ projection. reported pioneering data from experiments in which volumes, enthalpies, sound speeds, and temperatures were measured in iron wire which had been pulse Joule-heated to temperatures in the range from $2125 \mathrm{~K}$ to $3950 \mathrm{~K}$ at a pressure of $0.2 \mathrm{GPa}$ in argon gas. They give empirical expressions for $\rho, C_{P}$, and $V_{b}$, which allow an expression for $\gamma$ to be obtained

$$
\gamma=\frac{(0.5905728 \rho-792.7509)^{2}}{815.37 \rho}
$$

for $\rho$ in $\mathrm{kg} / \mathrm{m}^{3}$. Hixson et al. [1990] assign uncertainties of $20 \%$ to values of $\gamma$ thus obtained.

Hugoniot sound speed measurements. Brown and $\mathrm{Mc}$ Queen [1986] report high-quality data for the compressional sound speed $V_{P}$ in shocked iron. For liquids, $V_{p}=V_{b}$, so that $(\partial E / \partial P)_{V}$ is also obtained

$$
(\partial E / \partial P)_{V}=\frac{U^{\prime} \eta^{2}}{\rho_{00}}\left[1+U^{\prime} \eta+R^{* 2}\left(U^{\prime} \eta-1\right)\right]^{-1}=V / \gamma
$$

where $R^{*}=\rho V_{p} / \rho_{00} U_{s}, \eta=u_{p} / U_{s}$, and $U^{\prime}=d U_{s} / d u_{p}$. We have used our new values for $C_{0}, s$, and $s^{\prime}$ to reevaluate the sound speeds and the resulting values of $\gamma$ (Table 2) reported by Brown and McQueen [1986].

Shock wave equation of state data. SWEOS experiments yield measurements of $\rho, P$, and $E$ along the shock Hugoniot curve. Usually, $E_{t r}, \gamma$, and $K_{S}$ and its pressure derivatives are fit to the data by equating the energy increase during the shock process with that obtained via a transformation from

Table 2. High-Pressure Sound Speeds and Grüneisen Parameters

\begin{tabular}{cccc}
\hline$\rho_{0}, \mathrm{~kg} / \mathrm{m}^{3}$ & $P, \mathrm{GPa}$ & $V_{b}, \mathrm{~m} / \mathrm{s}$ & $\gamma$ \\
\hline $12643 \pm 353$ & $278.3 \pm 8.5$ & $9546 \pm 82$ & $1.419 \pm 0.272$ \\
$(12540 \pm 110)$ & $(275 \pm 8)$ & $(920 \pm 70)$ & $(1.564 \pm 0.123)$ \\
$13015 \pm 81$ & $331.2 \pm 2.4$ & $9985 \pm 87$ & $1.401 \pm 0.265$ \\
$(12920 \pm 40)$ & $(333 \pm 2)$ & $(10190 \pm 40)$ & $(1.580 \pm 0.065)$ \\
$13417 \pm 84$ & $396.7 \pm 2.8$ & $10622 \pm 86$ & $1.303 \pm 0.269$ \\
$(13280 \pm 40)$ & $(400 \pm 2)$ & $(10910 \pm 40)$ & $(1.506 \pm 0.060)$ \\
\hline
\end{tabular}

Both the original values presented by Brown and $\mathrm{MC}$ Queen [1986] (in parentheses) and the reevaluated values are presented. 


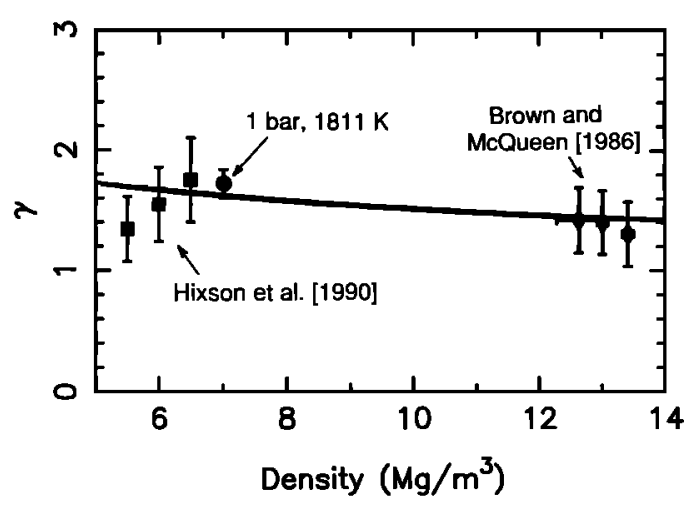

Figure 4. Data for thermodynamic Grüneisen parameter $\gamma$ compared with a fit using the assumption of $\gamma \propto \rho^{-n}$.

the experimental initial state to the phase on the Hugoniot, followed by isentropic compression to the shocked state volume $V_{H}$ and isochoric heating to the Hugoniot pressure. Assuming that the initial pressure is negligible, this is expressed as

$$
\frac{1}{2} P_{H}\left(V_{00}-V_{H}\right)=E_{S}+E_{t r}+V_{H} \int_{P_{S}}^{P_{H}} \frac{1}{\gamma} d P
$$

where the subscripts $H$ and $S$ refer to states on the Hugoniot and reference isentrope at volume $V_{H}$, respectively.

Unfortunately, the fit is relatively insensitive to $\gamma$, since a number of other parameters are also being fit. Also, $\gamma$ only enters the fit as an integral quantity, so any one experiment gives only a weighted average of $\gamma$ over a range of conditions. Although data from initially porous samples (i.e., with a larger $V_{00}$ ) are sometimes used to get results which are more sensitive to $\gamma$, this approach still yields only an integral quantity. In addition, the general quality of data from porous samples makes the fitting process rather subjective, as illustrated by the results for $\gamma$ obtained by McQueen et al. [1970] and Jeanloz [1979], who used the same data set to arrive at rather different conclusions concerning the behavior of $\gamma$. Finally, the phase on the porous Hugoniot is not well known, so there is a danger of including solid state results. Given these problems, we have chosen to exclude SWEOS data from our fit for $\gamma$ and rely on the other types of data already discussed.

Fitting of $\gamma$. Often the assumption is made that $\gamma \propto \rho^{-n}$. However, a number of authors (see, for example, Mulargia [1977], Stacey [1981], Falzone and Stacey [1981]) have been critical of this assumption. Examination of Figure 4 shows that this assumption, which does not allow the sign of $d \gamma / d \rho$ to change, does not accurately describe the available data.

We have chosen to evaluate several different functional forms for $\gamma$ with a maximum of three adjustable parameters and using $\rho, E$, and $P$ as independent variables. Data used for the fit (Table 3) are values of $\gamma$ calculated from (12) for $\rho$ $=5500,6000$, and $6500 \mathrm{~kg} / \mathrm{m}^{3}$ to represent the data of Hixson et al. [1990], along with the values of $\gamma$ obtained from the reference state properties and the reevaluated data of Brown and McQueen [1986].

Table 4 gives the functional forms evaluated and the results of the fitting process. In most cases the forms are simple parametric expressions not chosen because of any special physical significance. The expressions which allow explicit dependence on only one variable are less satisfactory than the majority of the two-variable fits. A dependence on $P$ alone is not studied, since the data of Hixson et al. [1990] showed a definite variation in $\gamma$ at constant pressure. Most of the two-variable fits give values of $\chi^{2}$ in the range 0.5 to 0.6 . One form, namely

$$
\gamma=\gamma_{0}+\gamma^{\prime}\left(\rho / \rho_{0}\right)^{n}\left(E-E_{0}\right)
$$

where $\gamma_{0}=1.732 \pm 0.111, \gamma^{\prime}=-0.136 \pm 0.082 \mathrm{~kg} / \mathrm{MJ}$, and $n=-1.642 \pm 0.917$, gives a significantly better fit $\left(\chi^{2}=\right.$ $0.13)$ than any other form.

The last form presented in Table 4 derives from the equation of state proposed by Tillotson [1962] for metals in the high-pressure and high-temperature regime associated with hypervelocity impact. Aside from (15), this expression gives the best fit to the data. While it might be argued that the equation of state proposed by Tillotson [1962] should be adopted here, it still gives a worse fit than the expression in (15), which we will retain as the preferred form for $\gamma$.

There is also a question of whether it is appropriate to use a single expression for $\gamma$ in both the compressed and expanded states represented by the data, especially since the liquidus solid phase at high pressure is different from that at low pressure. However, phase changes in the solid arise because of the detailed energetics of atomic spacings in different periodic lattice configurations with a given interatomic potential. A solid phase change occurs because the average interatomic distance has changed enough that a different lattice is more energetically favorable. The absence of periodicity in the structure of liquids precludes such sudden changes of state. Liquid state structural changes do occur and are reflected in our calculations (see Appendix A), but the change is smooth and continuous, justifying the use of a single functional form for $\gamma$ and also for the specific heat.

Equation (15) implies a temperature dependence for $\gamma$.

Table 3. Experimentally Constrained Values of the Thermodynamic Grüneisen Parameter

\begin{tabular}{ccccl}
\hline$P, \mathrm{GPa}$ & $\rho, \mathrm{kg} / \mathrm{m}^{3}$ & $E-E_{0}, \mathrm{MJ} / \mathrm{kg}$ & $\gamma$ & \\
\hline $10^{-4}$ & $7019 \pm 2$ & 0 & $1.723 \pm 0.116$ & this work \\
0.2 & 5500 & $1.8116 \pm 0.0634$ & $1.344 \pm 0.269$ & Hixson et al. [1990] \\
0.2 & 6000 & $1.1876 \pm 0.0511$ & $1.547 \pm 0.309$ & Hixson et al. [1990] \\
0.2 & 6500 & $0.5631 \pm 0.0391$ & $1.751 \pm 0.350$ & Hixson et al. [1990] \\
$277.4 \pm 18.4$ & $12643 \pm 353$ & $5.419 \pm 0.333$ & $1.419 \pm 0.272$ & Brown and McQueen [1986] \\
$331.5 \pm 9.4$ & $13015 \pm 81$ & $7.071 \pm 0.070$ & $1.401 \pm 0.265$ & Brown and McQueen [1986] \\
$397.1 \pm 10.2$ & $13417 \pm 84$ & $9.184 \pm 0.084$ & $1.303 \pm 0.269$ & Brown and McQueen [1986] \\
\hline
\end{tabular}


Table 4. Functional Forms and Fitting Results for $\gamma$

\begin{tabular}{|c|c|c|c|c|}
\hline & Form & & Fit Parameters & $\chi^{2}$ \\
\hline$\gamma_{0}\left(\rho / \rho_{0}\right)^{n}$ & & & $\begin{array}{l}\gamma_{0}=1.619 \pm 0.089 \\
n=-0.196 \pm 0.176\end{array}$ & 3.02 \\
\hline$\gamma_{0}+\gamma^{\prime}(E-$ & $\left.-E_{0}\right)$ & & $\begin{array}{l}\gamma_{0}=1.684 \pm 0.101 \\
\gamma^{\prime}=-0.0454 \pm 0.0255 \mathrm{~kg} / \mathrm{MJ}\end{array}$ & 1.21 \\
\hline$\gamma_{0}+\gamma^{\prime}\left(\rho / \rho_{0}\right)$ & )$^{n}\left(E-E_{0}\right)$ & & $\begin{array}{l}\gamma_{0}=1.732 \pm 0.111 \\
\gamma^{\prime}=-0.136 \pm 0.082 \mathrm{~kg} / \mathrm{MJ} \\
n=-1.642 \pm 0.917\end{array}$ & 0.13 \\
\hline$\gamma_{0}+\gamma^{\prime}\left(\rho / \rho_{0}\right)$ & & & $\begin{array}{l}\gamma_{0}=1.657 \pm 0.097 \\
\gamma^{\prime}=-5.52( \pm 87.09) \times 10^{-5} \mathrm{GPa}^{-1} \\
n=4.33 \pm 24.99\end{array}$ & 1.90 \\
\hline$\left(\rho / \rho_{0}\right)^{n}\left[\gamma_{0}+\right.$ & $\left.\gamma^{\prime}\left(E-E_{0}\right)\right]$ & & $\begin{array}{l}\gamma_{0}=1.707 \pm 0.105 \\
\gamma^{\prime}=-0.0747 \pm 0.0441 \mathrm{~kg} / \mathrm{MJ} \\
n=0.278 \pm 0.384\end{array}$ & 0.62 \\
\hline$\left(\rho / \rho_{0}\right)^{n}\left(\gamma_{0}+\right.$ & $\left.\boldsymbol{\gamma}^{\prime} \boldsymbol{P}\right)$ & & $\begin{array}{l}\gamma_{0}=1.703 \pm 0.104 \\
\gamma^{\prime}=-0.00216 \pm 0.00110 \mathrm{GPa}^{-1} \\
n=0.567 \pm 0.534\end{array}$ & 0.70 \\
\hline$\gamma_{0}\left(\rho / \rho_{0}\right)^{n}+$ & $\gamma^{\prime}\left(E-E_{0}\right)$ & & $\begin{array}{l}\gamma_{0}=1.715 \pm 0.107 \\
\gamma^{\prime}=-0.0898 \pm 0.0617 \mathrm{~kg} / \mathrm{MJ} \\
n=0.277 \pm 0.318\end{array}$ & 0.51 \\
\hline$\gamma_{0}\left(\rho / \rho_{0}\right)^{n}+$ & $\gamma^{\prime} P$ & & $\begin{array}{l}\gamma_{0}=1.709 \pm 0.106 \\
\gamma^{\prime}=-0.00343 \pm 0.00294 \mathrm{GPa}^{-1} \\
n=0.640 \pm 0.587\end{array}$ & 0.59 \\
\hline$\gamma_{0}+\gamma_{1}^{\prime} P+$ & $\gamma_{2}^{\prime}\left(E-E_{0}\right)$ & & $\begin{array}{l}\gamma_{0}=1.715 \pm 0.108 \\
\gamma_{1}^{\prime}=0.00235 \pm 0.00277 \mathrm{GPa}^{-1} \\
\gamma_{2}^{\prime}=-0.155 \pm 0.131 \mathrm{~kg} / \mathrm{MJ}\end{array}$ & 0.49 \\
\hline$\gamma_{0}+\gamma_{1}^{\prime}\left(\rho / \rho_{0}\right)$ & $+\gamma_{2}^{\prime}\left(E-E_{0}\right)$ & & $\begin{array}{l}\gamma_{0}=1.287 \pm 0.497 \\
\gamma_{1}^{\prime}=0.425 \pm 0.522 \\
\gamma_{1}^{\prime}=0.425 \pm 0.522 \\
\gamma_{2}^{\prime}=-0.0957 \pm 0.0667 \mathrm{~kg} / \mathrm{MJ}\end{array}$ & 0.55 \\
\hline$\gamma_{0}+\gamma_{1}^{\prime}\left(\rho / \rho_{0}\right)$ & $+\gamma_{2}^{\prime} P$ & & $\begin{array}{l}\gamma_{0}=0.580 \pm 0.980 \\
\gamma_{1}^{\prime}=1.128 \pm 1.020 \\
\gamma_{2}^{\prime}=-0.00383 \pm 0.00275 \mathrm{GPa}^{-1}\end{array}$ & 0.66 \\
\hline$a+\left(\gamma_{0}-a\right)$ & {$\left[\frac{E-E_{0}}{A}\left(\rho_{0} / \rho\right)^{2}+1\right]$} & & $\begin{array}{l}\gamma_{0}=1.730 \pm 0.115 \\
a=-3.665 \pm 181.3 \\
A=68.69 \pm 2435 \mathrm{MJ} / \mathrm{kg}\end{array}$ & 0.29 \\
\hline
\end{tabular}

Mulargia [1977] pointed out that there are good reasons to expect that $\gamma$ will vary as a function of temperature at constant volume, and from (15) we can see that

$$
(\partial \gamma / \partial T)_{V}=\gamma^{\prime}\left(\rho / \rho_{0}\right)^{n} C_{V}
$$

Pressure derivatives of $K_{S}$. To fit the pressure derivatives of $K_{S 0}\left(K_{S 0}^{\prime}=\left.\left(\partial K_{S} / \partial P\right)_{S}\right|_{P o, T o}\right.$ and $K_{S 0}^{\prime \prime}=\left(\partial^{2} K_{S} l\right.$ $\left.\left.\partial P^{2}\right)\left._{S}\right|_{P o, T o}\right)$, we can employ any of several compression curve formulations [Anderson, 1967; Stacey et al., 1982; Hofmeister, 1991], each giving the variation of $P$ as a function of density. The variations between different expressions are equivalent to differences in the form assumed for the interatomic potential. Because the Birch-Murnaghan finite strain equation of state is very successful in describing the compression of a large number of materials, we use it in this study. The pressure on the reference isentrope is given by

$$
\begin{gathered}
P_{S}=\frac{3}{2} K_{S 0}\left(x^{7}-x^{5}\right)\left[1+\xi_{1}-\xi_{1} x^{2}+\xi_{2}\left(x^{2}-1\right)^{2}\right] \\
x=\left(\rho / \rho_{0}\right)^{1 / 3} \\
\xi_{1}=3\left(4-K_{S 0}^{\prime}\right) / 4
\end{gathered}
$$

$$
\xi_{2}=\frac{3}{8} K_{s 0} K_{s 0}^{\prime \prime}+\frac{3}{8} K_{s 0}^{\prime}\left(K_{s 0}^{\prime}-7\right)+\frac{143}{24}
$$

where $V_{0}$ is the specific volume at $P_{0}$ and $T_{0}$. The form given is fourth order in strain or, if $\xi_{2}=0$, third order. The change in the internal energy $E_{S}$ along the isentrope [Ahrens, 1979] is

$$
\begin{aligned}
E_{S}=\frac{9}{2} V_{0} K_{S 0}\left[\left(\xi_{1}\right.\right. & +1)\left(\frac{x^{4}}{4}-\frac{x^{2}}{2}+\frac{1}{4}\right)-\xi_{1}\left(\frac{x^{6}}{6}-\frac{x^{4}}{4}+\frac{1}{12}\right) \\
& \left.+\xi_{2}\left(\frac{x^{8}}{8}-\frac{x^{6}}{2}+\frac{3 x^{4}}{4}-\frac{x^{2}}{2}+\frac{1}{8}\right)\right]
\end{aligned}
$$

$K_{S 0}^{\prime}$ and $K_{S 0}^{\prime \prime}$ are obtained by fitting the shock Hugoniot data to a rearrangement of (14), supplying the (already constrained) values of $E_{t r}, K_{s 0}, \rho_{0}$, and the results for $\gamma$.

Use of (15) for $\gamma$ complicates evaluation of the integral in (14) somewhat. Let us rearrange $d E=V d P / \gamma$ to $\gamma d E=V d P$ so that 


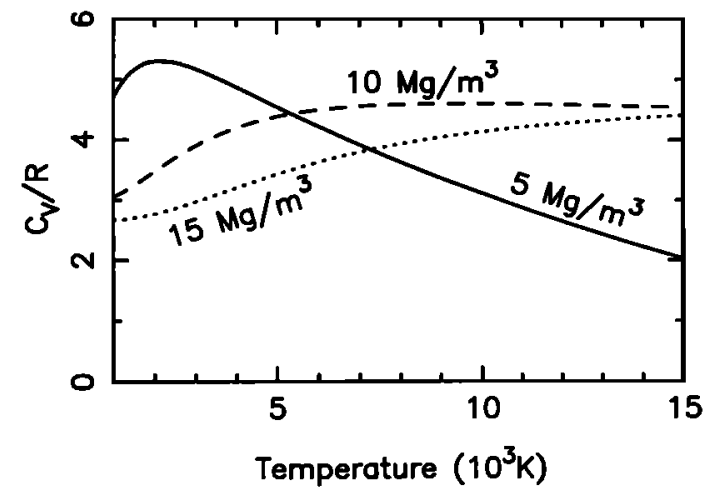

Figure 5. Constant volume specific heat as a function of temperature predicted by the present model for three different densities. The decrease at high temperatures for the lowest density reflects the essentially uniform spatial distribution at those conditions.

$$
\int_{E_{1}}^{E_{2}} \gamma d E=\int_{E_{1}}^{E_{2}}\left[\gamma_{0}+\gamma^{\prime}\left(\rho / \rho_{0}\right)^{n} E\right] d E=V \int_{P_{1}}^{P_{2}} d P
$$

Evaluating the integrals, we get

$$
\gamma_{0}\left(E_{2}-E_{1}\right)+\frac{1}{2} \gamma^{\prime}\left(\rho / \rho_{0}\right)^{n}\left(E_{2}^{2}-E_{1}^{2}\right)=V\left(P_{2}-P_{1}\right)
$$

Assuming $\boldsymbol{P}_{0}$ is negligible, we can write this in terms of known quantities by setting $E_{2}=E_{H}-E_{t r}, E_{1}=E_{S}, P_{1}$ $=P_{S}, P_{2}=P_{H}$, and $V=V_{H}$. Since $E_{H}=\frac{1}{2} P_{H}\left(V_{00}-\right.$ $\left.V_{H}\right)$ we get

$$
\begin{aligned}
V_{H}\left(P_{H}\right. & \left.-P_{S}\right)=\gamma_{0}\left[\frac{1}{2} P_{H}\left(V_{00}-V_{H}\right)-E_{t r}-E_{S}\right] \\
& +\frac{1}{2} \gamma^{\prime}\left(\rho / \rho_{0}\right)^{n}\left\{\left[\frac{1}{2} P_{H}\left(V_{00}-V_{H}\right)-E_{t r}\right]^{2}-E_{S}^{2}\right\}
\end{aligned}
$$

This can be rearranged to give

$$
\begin{gathered}
P_{H}=\frac{-b-\left(b^{2}-4 a c\right)^{1 / 2}}{2 a} \\
a=\frac{1}{8} \gamma^{\prime}\left(\rho / \rho_{0}\right)^{n}\left(V_{00}-V_{H}\right)^{2} \\
c=\frac{1}{2}\left[\gamma_{0}-\gamma^{\prime}\left(\rho / \rho_{0}\right)^{n} E_{t r}\right]\left(V_{00}-V_{H}\right)-V_{H} \\
c=\left[\frac{1}{2} \gamma^{\prime}\left(\rho / \rho_{0}\right)^{n}\left(E_{t r}-E_{S}\right)-\gamma_{0}\right]\left(E_{t r}+E_{S}\right)+P_{S} V_{H}
\end{gathered}
$$

$K_{S 0}^{\prime}$ and $K_{S 0}^{\prime \prime}$ enter the solution through $E_{S}$ and $P_{S}$ in the coefficient $c$.

Knowledge of $K_{S 0}^{\prime}$ and $K_{s 0}^{\prime \prime}$ allows the $U_{s}-u_{p}$ Hugoniot curve to be calculated as well. Since we wish our final model to be internally consistent the new $U_{s}-u_{p}$ relation is used to refine the analysis of the Hugoniot sound speed data and obtain a refined estimate of parameters for $\gamma$, which in turn may be used with the SWEOS data to refine $K_{S 0}^{\prime}$ and $K_{S 0}^{\prime \prime}$. We iterate this process until convergence is achieved, preferentially minimizing $\chi^{2}$ for the $P-V$ Hugoniot data, since the available data constrain the $P-V$ Hugoniot better than they constrain $\gamma$. The final model gives $\gamma_{0}=1.735 \pm 0.111$, $\gamma^{\prime}=-0.130 \pm 0.080 \mathrm{~kg} / \mathrm{MJ}, n=-1.870 \pm 0.974, K_{s 0}^{\prime}=$ $4.661 \pm 0.040$, and $K_{S 0}^{\prime \prime}=-0.043 \pm 15.3 \mathrm{GPa}^{-1}$, with $\chi^{2}$ $=0.13$ for $\gamma$ and $\chi^{2}=18.72$ for $P_{H}$. The large uncertainty in
$K_{s 0}^{\prime \prime}$ requires us to test the significance of the fourth-order Birch-Murnaghan fit. Initial fits with the original parameters for $\gamma$ give $\chi^{2}=29.3$ and 20.3 for the third- and fourth-order fits, respectively, so that the standard $F$ statistic is 3.55 . Application of the $F$ test for significance of the fourth-order term shows that $F>3.23$ indicates that the extra term is justified at the $95 \%$ confidence level. The uncertainty in $K_{S 0}^{\prime \prime}$ is simply a result of its covariance with $K_{S 0}^{\prime}$. The final model gives $\alpha_{0}=9.27( \pm 0.85) \times 10^{-5} \mathrm{~K}^{-1}$ and $\left.(\partial \gamma / \partial T)_{V}\right|_{1 \mathrm{bar}, 1811 \mathrm{~K}}$ $=-8.4( \pm 5.2) \times 10^{-5} \mathrm{~K}^{-1}$.

Specific heat. The constant volume specific heat $C_{V}=$ $(\partial E / \partial T)_{V}$ is required to complete the equation of state. Reliable data are lacking for the behavior of $C_{V}$ at high pressures, so we must appeal to theoretical techniques. The specific heat may be expressed as the sum

$$
C_{V}=C_{k}+C_{p o t}+C_{e}
$$

where the subscripts $k$, pot, and $e$ denote the kinetic contribution from thermal motion of the ionic cores, the potential energy contribution from the ionic cores, and the energy due to the electronic density of states, respectively. For $C_{k}$ we use the high temperature limit $C_{k}=1.5 R / \mu$, where $\mu$ is the atomic weight and $R$ is the ideal gas constant. Development of $C_{p o t}$ and $C_{e}$, however, is more involved. In Appendix A we describe liquid structure calculations used to obtain $C_{p o t}$ and use a parameterization of the results of Boness et al. [1986] for $\varepsilon$-Fe to model $C_{e}$. Figure 5 shows results of our $C_{V}$ model. The upper limit to $C_{V}$ for liquid $\mathrm{Fe}$ is about 5.3 R, with $C_{V} \approx 4.0 \mathrm{R}$ to $4.5 \mathrm{R}$ for $\rho-T$ conditions of geophysical interest.

\section{Discussion and Comparison With Other Equations of State}

Appendix C summarizes the final EOS parameters. The principal $U_{s}-u_{p}$ Hugoniot given by these parameters requires a quadratic expression with $C_{0}=3694.5 \mathrm{~m} / \mathrm{s}, s=$ 1.7254 , and $s^{\prime}=-2.571 \times 10^{-5} \mathrm{~s} / \mathrm{m}$. Figure 6 shows the principal shock Hugoniot predicted by the present model
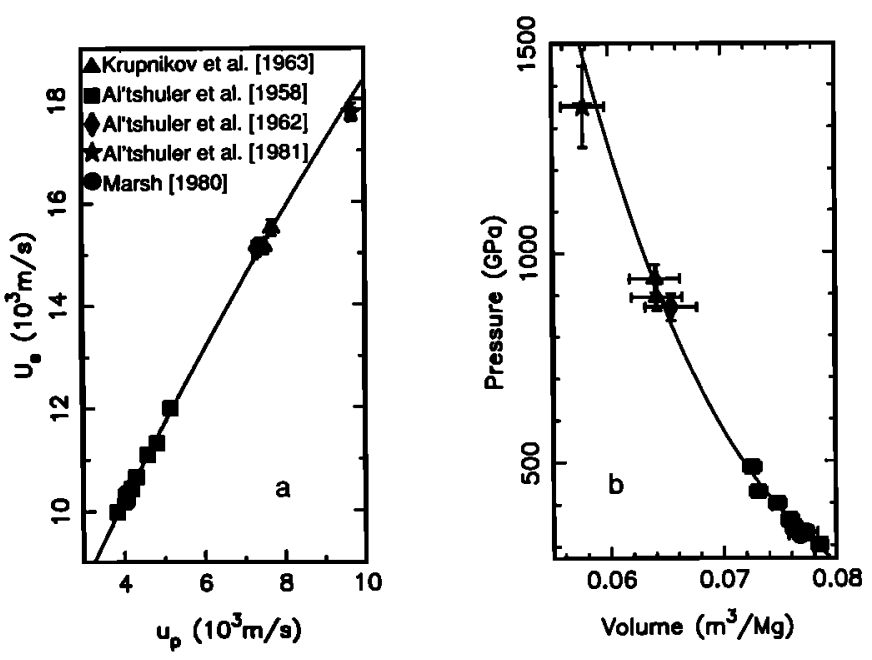

Figure 6. Principal Hugoniot of nonporous Fe predicted by final EOS model and compared with existing data in liquid Fe stability region. (a) $U_{s}-u_{p}$ projection. (b) $P-V$ projection. 


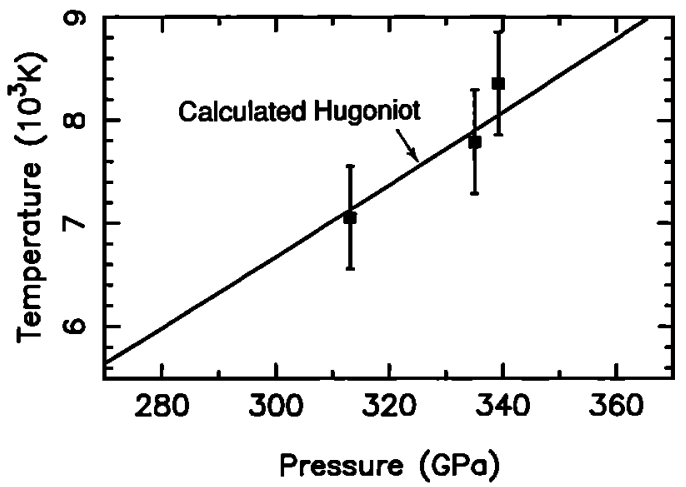

Figure 7. Predicted $P-T$ Hugoniot for Fe with data from Yoo et al. [1993].

EOS compared with all available data. Agreement with the data is quite good, even falling within the uncertainty of the highest pressure datum, which had been excluded because of its apparent disagreement with the trend established in $U_{s}-$ $u_{p}$ space by the other data.

Although we chose not to constrain our model with data for temperatures $T_{H}$ on the Hugoniot of $\mathrm{Fe}$, we do wish to compare values of $T_{H}$ predicted by our model with the data. For temperatures in the liquid $\mathrm{Fe}$ stability region the only data set is that of Yoo et al. [1993]. Ahrens et al. [1990] have shown that the other data set, that of Bass et al. [1987], does not extend to high enough pressures to give data for completely liquid Hugoniot states. Figure 7 shows the calculated $P-T$ Hugoniot with the data from Yoo et al. [1993]. Our model predicts values of $T_{H}$ which are in excellent agreement with the data.

Previous EOS Results and Shock Compression of Porous Fe

Figures 8 and 9 compare the data of McQueen et al. [1970] to Hugoniot curves calculated for porous Fe by using the present equation of state. The predicted curves are in excellent agreement with the data. The agreement at lower pressures is somewhat surprising, since the Hugoniot states in these regions are not completely liquid. Also shown are Hugoniot curves predicted by McQueen et al. [1970] and Jeanloz [1979]. Both of these previous efforts used SWEOS data for all but the most porous samples to constrain $\gamma$, assuming $\gamma \propto \rho^{-n}$. For the high-pressure/high-velocity portions of the Hugoniot curves, where we expect the liquid to be the stable phase, the present model compares favorably with the previous models. The present model exhibits interesting behavior on the Hugoniot curve for the most porous samples (average $\rho_{00} \approx 3368 \mathrm{~kg} / \mathrm{m}^{3}$ ). Below $P \approx 38$ GPa the solution of (25) is complex, indicating that states in liquid Fe for $P<38 \mathrm{GPa}$ cannot be reached via a steady shock wave from an initial density of $3368 \mathrm{~kg} / \mathrm{m}^{3}$. The present model still gives thermodynamic states for this pressure range, but they cannot be achieved via the RankineHugoniot equations from a very low initial density. In any case, the lower pressure data are probably not in the completely liquid stability region, so that the present model is not appropriate for calculating these states.

\section{Implications for Earth's Core}

Figure 10 shows temperatures, densities, isentropic bulk moduli, and sound velocities computed from the present model for isentropes having temperatures of $5000 \mathrm{~K}, 6000 \mathrm{~K}$, $7000 \mathrm{~K}$, and $8000 \mathrm{~K}$ at the inner core boundary (ICB) pressure of $329 \mathrm{GPa}$ [Dziewonski and Anderson, 1981]. Comparison with the preliminary reference Earth model (PREM) [Dziewonski and Anderson, 1981] provides information on both the behavior of alloying elements in the core and the sensitivity of core composition models to assumptions.

The first important note concerns the sensitivity of the density of liquid Fe to the temperature. The four isentropes
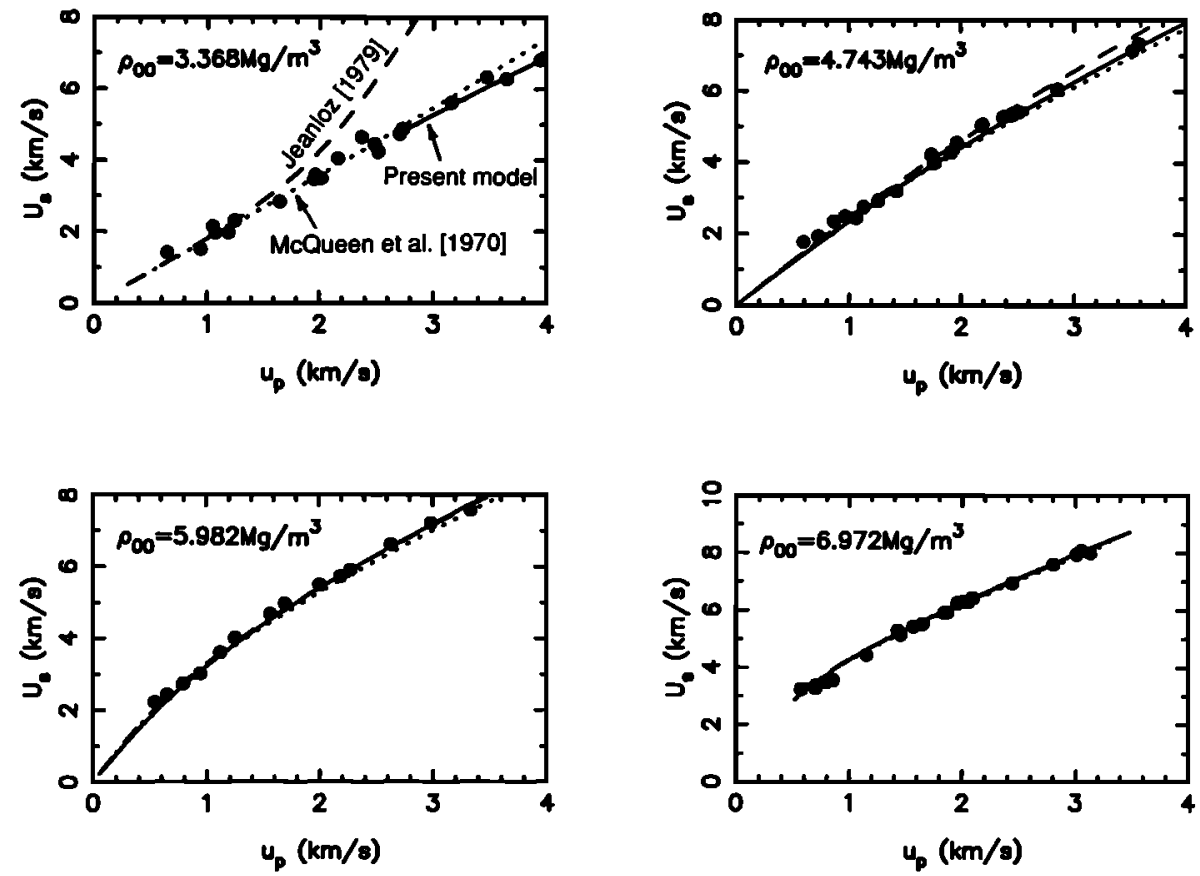

Figure 8. $U_{s}-u_{p}$ Hugoniot curves for initial porous Fe. Data are from McQueen et al. [1970]. 

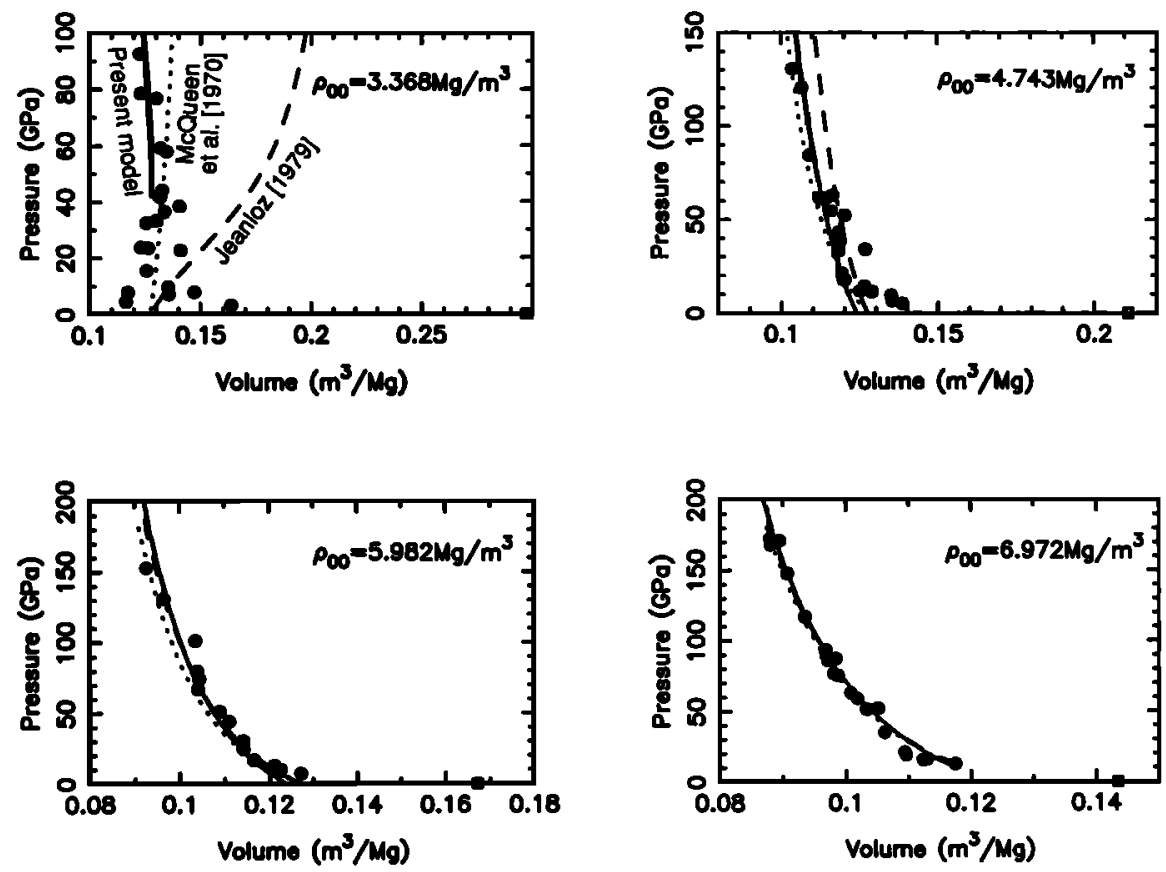

Figure 9. As in Figure 8 but $P-V$ Hugoniot curves are shown. Lower pressure regions are not in liquid Fe stability field.

range from $5 \%$ to $10 \%$ more dense than the PREM outer core. We also note that the PREM inner core is also $3-6 \%$ less dense than pure liquid $\mathrm{Fe}$, lending support to the assertion by Jephcoat and Olsen [1987] that the inner core must also have a significant light element component.

The bulk moduli predicted by the present model agree well with that of the PREM outer core, indicating that alloying elements in the outer core have little effect on the compress- ibility of liquid Fe. The value of $d K_{S} / d P$, however, is $\sim 10 \%$ greater for pure liquid $\mathrm{Fe}$ than for the outer core, indicating that the alloying elements decrease the pressure dependence of $K_{S}$ in liquid Fe. The combination of a higher density and approximately equal $K_{S}$ results in pure liquid $\mathrm{Fe}$ having compressional sound velocities about $3 \%$ lower than those of the PREM outer core. The pure Fe isentropes are nearly indistinguishable from one another in sound velocity, indi-
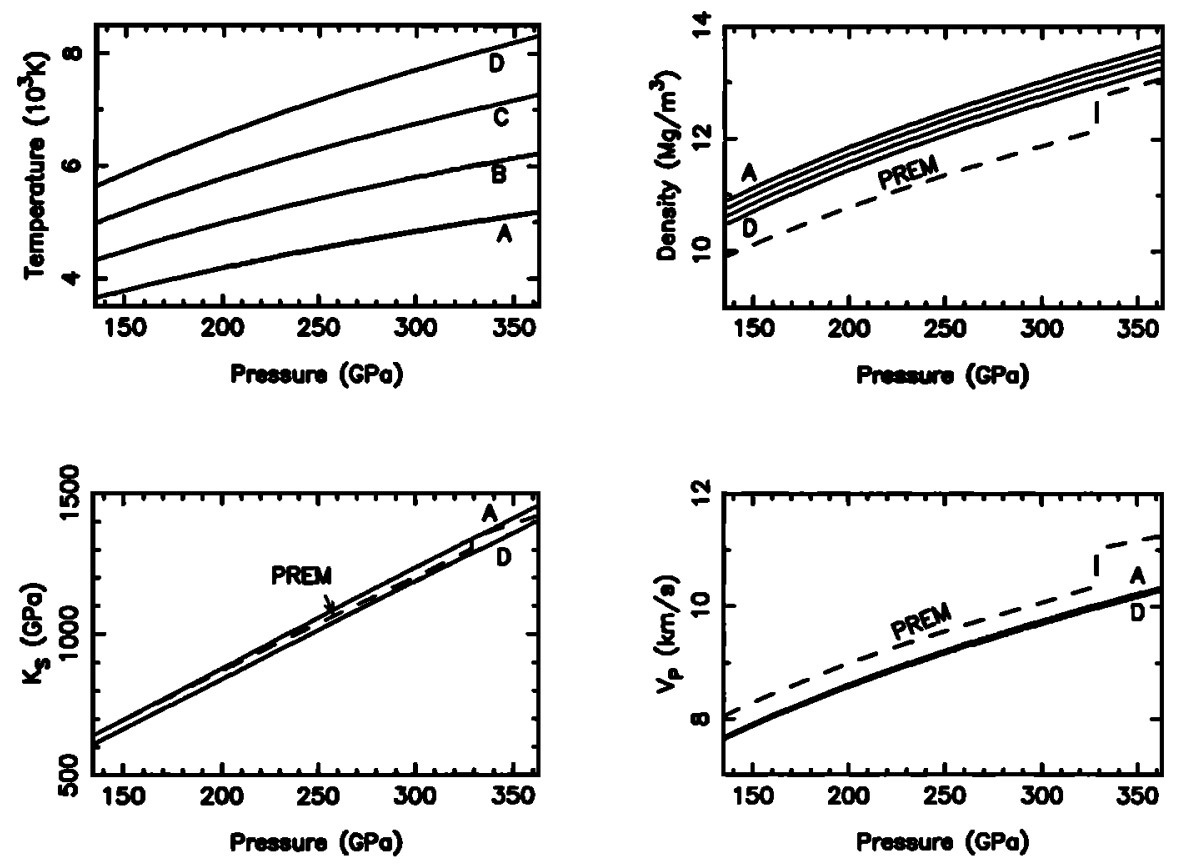

Figure 10. Properties of pure liquid Fe (solid lines) along isentropes having inner core boundary temperatures of (A) $5000 \mathrm{~K}$, (B) $6000 \mathrm{~K}$, (C) $7000 \mathrm{~K}$, and (D) $8000 \mathrm{~K}$. Curves for the PREM core are presented as dashed lines. 
cating that care must be taken when inverting seismic wave travel times for both $K_{S}$ and $\rho$ in the core.

\section{Summary}

We have combined a wide range of data and theoretical techniques to obtain the EOS parameters and functional form for $\gamma$ given in Appendix C. A notable result is a thermodynamic Grüneisen parameter $\gamma$ that depends explicitly on both density and internal energy. The model also gives $\alpha_{0}=9.27 \times 10^{-5} \mathrm{~K}^{-1}$ and $\left.(\partial \gamma / \partial T)_{V}\right|_{1 \mathrm{bar}, 1811 \mathrm{~K}}=-8.4$ $( \pm 5.2) \times 10^{-5} \mathrm{~K}^{-1}$.

Predicted Hugoniot curves show good agreement with shock wave equation of state data from porous Fe samples. The present equation of state model compares favorably in this respect with past models which have been developed assuming that $\gamma$ is solely density dependent.

Isentropes calculated with this model can be compared with seismological Earth models such as PREM to obtain information on the properties of other elements alloyed with $\mathrm{Fe}$ in the core. However, a priori assumptions have a significant effect on the outcomes of such comparisons. The density deficit (and thus concentration of light elements) in the outer core is strongly dependent upon the assumed temperature of the core. The present results indicate that the outer core is $5 \%\left(T_{I C B}=8000 \mathrm{~K}\right)$ to $10 \%\left(T_{I C B}=5000 \mathrm{~K}\right)$ less dense than pure Fe. The inner core is $3-6 \%$ less dense, supporting arguments for a light component in the inner core as well. We find that alloying elements in the core do not significantly affect the magnitude of the bulk modulus of liquid Fe but may cause the pressure dependence of the bulk modulus to decrease. Combination of the calculated bulk modulus and density shows that sound speeds in liquid Fe are nearly independent of temperature at core pressures, suggesting that an important test for candidate core compositions is the effects of alloying elements on the sound speed of liquid $\mathrm{Fe}$, which is also one of the most directly observable properties of the outer core.

\section{Appendix A: Potential and Electronic Energy Contributions to $C_{V}$}

\section{Electronic Energy Term}

The functional form of $C_{e}$ is probably similar for all condensed phases of $\mathrm{Fe}$ [Boness et al., 1986; Weir et al., 1983], although Hausleitner and Hafner [1988] note that liquid transition metals seem to show more s-d hybridization than the solid phases. We use $C_{e}$ for $\varepsilon$-Fe [Boness et al., 1986], which we fit with a single analytic expression

$$
\begin{gathered}
C_{e}=A\left(1-\frac{\Theta^{2}}{\Theta^{2}+T^{2}}\right)+B T^{3 / 5} \\
A=A_{0}+A_{1} \rho_{0} / \rho \\
B=B_{0}+B_{1}\left(\rho_{0} / \rho\right)^{2} \\
\Theta=\Theta_{0}\left(\rho / \rho_{0}\right)^{z}
\end{gathered}
$$

where $A_{0}=248.92 \mathrm{~J} \mathrm{~kg}^{-1} \mathrm{~K}^{-1}, A_{1}=289.48 \mathrm{~J} \mathrm{~kg}^{-1} \mathrm{~K}^{-1}$, $B_{0}=0.4057 \mathrm{~J} \mathrm{~kg}^{-1} \mathrm{~K}^{-8 / 5}, B_{1}=-1.1499 \mathrm{~J} \mathrm{~kg}^{-1} \mathrm{~K}^{-8 / 5}, \Theta_{0}$ $=1747.3 \mathrm{~K}$, and $z=1.537$. Jank et al. [1991] obtain a theoretical electronic density of states for liquid $\mathrm{Fe}$ which gives somewhat lower values for $\mathrm{Ce}$ but is otherwise in substantial agreement with the results of Boness et al. [1986]. The electronic contribution to the change in internal energy and entropy arising from a temperature change from $T_{1}$ to $T_{2}$ is

$$
\begin{aligned}
& \left(E_{2}-E_{1}\right)_{e}=\int_{T_{1}}^{T_{2}} C_{e} d T=A\left\{T_{2}-T_{1}-\Theta\left[\tan ^{-1}\left(T_{2} / \Theta\right)\right.\right. \\
& \left.\left.-\tan ^{-1}\left(T_{1} / \Theta\right)\right]\right\}+\frac{5}{8} B\left(T_{2}^{8 / 5}-T_{1}^{8 / 5}\right) \\
& \left(S_{2}-S_{1}\right)_{e}=\int_{T_{1}}^{T_{2}} \frac{C_{e}}{T} d T=A\left\{\ln \left(T_{2} / T_{1}\right)\right. \\
& \left.-\frac{1}{2} \ln \left[\frac{T_{2}^{2}\left(\Theta^{2}+T_{1}^{2}\right)}{T_{1}^{2}\left(\Theta^{2}+T_{2}^{2}\right)}\right]\right\}+\frac{5}{3} B\left(T_{2}^{3 / 5}-T_{1}^{3 / 5}\right)
\end{aligned}
$$

\section{Potential Energy Term}

For a liquid of spherical atoms having an interatomic potential $\varphi(r)$ with the distribution of atoms around a test atom described by a radial distribution function $g(r)$ and an atomic number density $n$, the total specific potential energy $E_{p o t}$ is

$$
E_{p o t}=2 \pi n \int_{0}^{\infty} \varphi(r) g(r) r^{2} d r
$$

We separate $\varphi(r)$ into an s-electron part $\varphi_{s}(r)$ and d-band bonding and repulsion terms $\left(\varphi_{b}(r)\right.$ and $\varphi_{r}(r)$, respectively):

$$
\varphi(r)=\varphi_{s}(r)+\varphi_{b}(r)+\varphi_{r}(r)
$$

According to Hausleitner and Hafner [1988] and Hafner and Heine [1986]

$$
\begin{gathered}
\varphi_{b}(r)=-2 Z_{d}\left(1-Z_{d} / 10\right)\left(12 / N_{c}\right)^{1 / 2}(28.06 / \pi) R_{d}^{3} / r^{5} \\
\varphi_{r}(r)=Z_{d}\left(450 / \pi^{2}\right) R_{d}^{6} / r^{8} \\
\varphi_{s}(r)=\frac{Z_{s}^{2}}{r}\left[\frac{1}{4} H\left(r-2 R_{c}\right)+\frac{1}{2} H(r)+\frac{1}{4} H\left(r+2 R_{c}\right)\right]
\end{gathered}
$$

where $Z_{d}$ and $Z_{s}$ are the number of $\mathrm{d}$ and $\mathrm{s}$ electrons per atom, $R_{c}$ is the pseudopotential empty core radius, $R_{d}$ is the d state radius, $N_{c}$ is the zero-point stable phase coordination number, and $H(r)$ is the real space screening function. Numerical factors in (A9) and (A10) are for energies in Rydbergs $\left(1 \mathrm{Ryd}=2.1797 \times 10^{-18} \mathrm{~J}\right)$ and distances in units of the Bohr radius, $a_{0}=5.29177 \times 10^{-11} \mathrm{~m} . H(r)$ is obtained using the treatment of Hafner and Heine [1986] with the Pettifor and Ward [1984] approximation to the normalized Lindhard susceptibility and the Ichimaru and Utsumi [1981] strongly coupled electron gas model.

The structure of a liquid is described by a set of equations relating $\varphi(r)$ to the correlation of atomic positions, which can be stated as

$$
\begin{array}{r}
h(r)=c(r)+n \int d \mathbf{r}^{\prime} h\left(\left|\mathbf{r}-\mathbf{r}^{\prime}\right|\right) c\left(\mathbf{r}^{\prime}\right) \\
c(r)=h(r)-\ln [g(r) \exp (\varphi(r) / k T)]+B(r)
\end{array}
$$




$$
g(r)=1+h(r)
$$

where $k$ is the Boltzmann constant [Rosenfeld and Ashcroft, 1979]. The variable $B(r)$ is generally not known a priori. The two basic approaches are to solve iteratively for a $B(r)$ which results in thermodynamic self-consistency or to choose a $B(r)$ that one has reason to believe is realistic for the case at hand. We take the second option, which is much simpler in practice. Rosenfeld and Ashcroft [1979], Lado et al. [1983], and Zerah and Hansen [1986] argue that $B(r)$ is the same set of functions for all potentials. We appeal to a weakened form of this assertion in the following manner. When we set $B=0$, we can reproduce the experimental $g(r)$ of Waseda and Suzuki [1970] for liquid Fe at the correct density but at the wrong temperature. We obtain $B(r)$ by taking the difference between $\varphi(r) / k T$ at the correct temperature $\left(1893 \mathrm{~K}\right.$ at $\left.6966 \mathrm{~kg} / \mathrm{m}^{3}\right)$ and at the temperature $(666.4$ K) required to give the observed first peak height and position in $g(r)$. Defining this difference to be $B(r)$ at $1893 \mathrm{~K}$ and $6966 \mathrm{~kg} / \mathrm{m}^{3}$, we require that $B(r)$ have the same dependence on temperature and packing fraction as $B(r)$ for a fluid of hard spheres with radii $r_{h s}=\left(r: \varphi(r)=\varphi_{\min }+3 k T / 2\right)$.

We solve (A12)-(A14) for $g(r)$ using the approach suggested by Gillan [1979] with the Press et al. [1986] implementation of the Newton-Raphson method. Energies are obtained by numerical integration of (A7). These energies are corrected for a baseline ambiguity, differentiated, and scaled to fit the observed values of $C_{p o t}$ (obtained by subtracting $C_{k}$ and $C_{e}$ from the published experimental result). The final results are fit with a simple analytic function of $T$ and $\rho$ to get $C_{p o r}$

$$
\begin{gathered}
C_{p o t}=\frac{\Lambda T+\Xi \theta}{\theta+T} \\
\Xi=\Xi_{0}\left(\rho_{0} / \rho\right)^{3 / 5} \\
\Lambda=\left[F\left(\Lambda_{0}+\Lambda_{1} \rho / \rho_{0}\right)+\Lambda_{2}\right]\left(\rho / \rho_{0}\right)^{2 / 5} \\
F=\frac{1}{1+e^{(\rho-D) / \Delta}}
\end{gathered}
$$

where $\theta=5000 \mathrm{~K}, \Lambda_{0}=-325.23 \mathrm{~J} \mathrm{~kg}^{-1} \mathrm{~K}^{-1}, \Lambda_{1}=302.07$ $\mathrm{J} \mathrm{kg}^{-1} \mathrm{~K}^{-1}, \Lambda_{2}=30.45 \mathrm{~J} \mathrm{~kg}^{-1} \mathrm{~K}^{-1}, \Xi_{0}=282.67 \mathrm{~J} \mathrm{~kg}^{-1}$ $\mathrm{K}^{-1}, D=7766 \mathrm{~kg} / \mathrm{m}^{3}$, and $\Delta=1146 \mathrm{~kg} / \mathrm{m}^{3}$. The contribution by this term to the change in internal energy and entropy with a temperature change from $T_{1}$ to $T_{2}$ is

$$
\begin{aligned}
& \left(E_{2}-E_{1}\right)_{p o t}=\int_{T_{1}}^{T_{2}} C_{p o t} d T=\Lambda\left(T_{2}-T_{1}\right) \\
& +\theta(\Xi-\Lambda) \ln \left(\frac{\theta+T_{2}}{\theta+T_{1}}\right) \\
& \left(S_{2}-S_{1}\right)_{p o t}=\int_{T_{1}}^{T_{2}} \frac{C_{p o t}}{T} d T=\Lambda \ln \left(\frac{\theta+T_{2}}{\theta+T_{1}}\right) \\
& +\Xi \ln \left[\frac{T_{2}\left(\theta+T_{1}\right)}{T_{1}\left(\theta+T_{2}\right)}\right]
\end{aligned}
$$

\section{Appendix B: Temperatures on the Reference Isentrope}

A quantity which is quite useful in models of core processes is the temperature on the reference isentrope centered at $P_{0}=1$ bar and $T_{0}=1811 \mathrm{~K}$. Since

$$
(\partial \ln T / \partial \ln \rho)_{S}=\gamma
$$

we get

$$
T_{S}=T_{0} \exp \left(\int_{\rho_{0}}^{\rho_{1}}(\gamma / \rho) d \rho\right)
$$

The solution of (B2) is particularly simple under the assumption that $\gamma$ is proportional to some power of $\rho$. Equation (B2) is still analytic and $E_{S}$ is given as a polynomial in $\rho$ by (21). Thus

$$
T_{S}=T_{0} \exp \left[\int_{\rho_{0}}^{\rho_{1}} \frac{\left[\gamma_{0}+\gamma^{\prime}\left(\rho / \rho_{0}\right)^{n} E_{S}\right]}{\rho} d \rho\right]
$$

or

$$
T_{S}=T_{0} \exp \left[\gamma_{0} \ln \left(\rho_{1} / \rho_{0}\right)+\gamma^{\prime} \rho_{0}^{-n} \int_{\rho_{0}}^{\rho_{1}} \rho^{n-1} E_{S}(\rho) d \rho\right]
$$

Evaluating the integral in (B4), we get

$$
\begin{aligned}
T_{S}=T_{0} \exp & \left\{\gamma_{0} \ln \left(\rho / \rho_{0}\right)+\frac{27}{2} \gamma^{\prime} V_{0} K_{S 0}\left[\frac { a _ { 1 } } { 3 n + 8 } \left(x^{3 n+8}\right.\right.\right. \\
-1)- & \frac{a_{2}}{3 n+6}\left(x^{3 n+6}-1\right)+\frac{a_{3}}{3 n+4}\left(x^{3 n+4}-1\right) \\
& \left.\left.\left.-\frac{a_{4}}{3 n+2}\left(x^{3 n+2}-1\right)+\frac{a_{5}}{3 n}\left(x^{3 n}-1\right)\right]\right\} \quad \text { (B5 }\right)
\end{aligned}
$$

where

$$
\begin{gathered}
a_{1}=\xi_{2} / 8 \\
a_{2}=\frac{\xi_{1}+3 \xi_{2}}{6} \\
a_{3}=\frac{1+2 \xi_{1}+3 \xi_{2}}{4} \\
a_{4}=\frac{1+\xi_{1}+\xi_{2}}{2} \\
a_{5}=\frac{6+4 \xi_{1}+3 \xi_{2}}{24}
\end{gathered}
$$

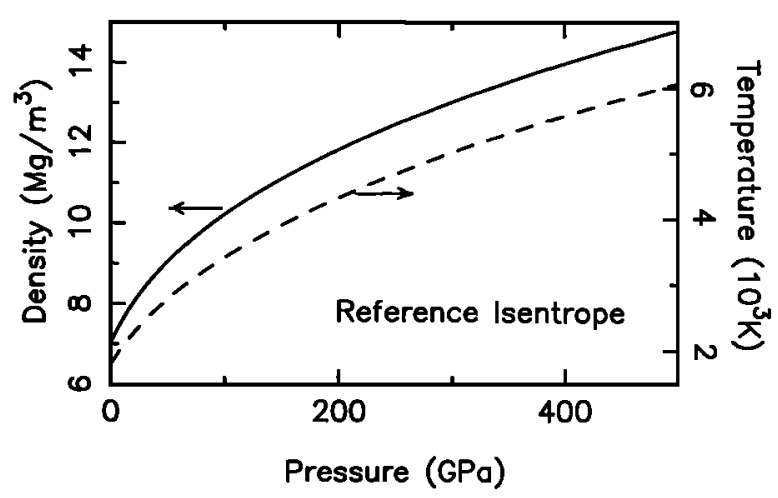

Figure B1. Variation in temperature and density along reference isentrope used in the present final EOS model. 
Figure B1 gives $\rho$ and $T$ as a function of $P$ along this reference isentrope.

\section{Appendix C: Best Fit Equation of State Parameters for Liquid Iron}

Reference Conditions

$$
\begin{aligned}
P_{0} & =1 \mathrm{bar}=10^{5} \mathrm{~Pa} \\
T_{0} & =1811 \mathrm{~K} \\
\rho_{0} & =7019 \pm 2 \mathrm{~kg} / \mathrm{m}^{3} \\
C_{P 0} & =835 \pm 54 \mathrm{~J} \mathrm{~kg}{ }^{-1} K^{-1} \\
E_{t r} & =E_{0}-E_{\alpha, 1 \mathrm{bar}, 298 \mathrm{~K}} \\
& =1.3007( \pm 0.0118) \times 10^{6} \mathrm{~J} / \mathrm{kg} \\
E_{0} & \equiv 0
\end{aligned}
$$

Reference Isentrope

Centered at $\left(P_{0}, T_{0}\right)$ :

$$
\begin{aligned}
& K_{S 0}=109.7 \pm 0.7 \mathrm{GPa} \\
& K_{S 0}^{\prime}=4.661 \pm 0.040 \\
& K_{S 0}^{\prime \prime}=-0.043 \pm 15.3 \mathrm{GPa}^{-1}
\end{aligned}
$$

Thermodynamic Grüneisen Parameter

$$
\begin{aligned}
\gamma & =\gamma_{0}+\gamma^{\prime}\left(\rho / \rho_{0}\right)^{n}\left(E-E_{0}\right) \\
\gamma_{0} & =1.735 \pm 0.111 \\
\gamma^{\prime} & =-0.130 \pm 0.080 \mathrm{~kg} / \mathrm{MJ} \\
n & =-1.870 \pm 0.974
\end{aligned}
$$

Constant Volume Specific Heat

$$
\begin{gathered}
C_{V}=C_{k}+C_{p o t}+C_{e} \\
C_{e}=A\left(1-\frac{\Theta^{2}}{\Theta^{2}+T^{2}}\right)+B T^{3 / 5} \\
A\left(\mathrm{~J} \mathrm{~kg}^{-1} K^{-1}\right)=248.92+289.48\left(\rho_{0} / \rho\right) \\
B\left(\mathrm{~J} \mathrm{~kg}^{-1} K^{-8 / 5}\right)=0.4057-1.1499\left(\rho_{0} / \rho\right)^{2} \\
\Theta(\mathrm{K})=1747.3\left(\rho / \rho_{0}\right)^{1.537} \\
C_{k}=223.32 \mathrm{~J} \mathrm{~kg} K^{-1} \\
C_{p o t}=\frac{\Lambda T+\Xi \theta}{\theta+T} \\
\theta=5000 \mathrm{~K} \\
\Xi\left(\mathrm{J} \mathrm{kg}^{-1} K^{-1}\right)=282.67\left(\rho_{0} / \rho\right)^{3 / 5} \\
\Lambda\left(\mathrm{J} \mathrm{kg}{ }^{-1}\right)=\left[F\left(302.07 \rho / \rho_{0}-325.23\right)\right. \\
+30.45]\left(\rho / \rho_{0}\right)^{2 / 5} \\
F=\frac{1}{1+e^{(\rho-D) / \Delta}}
\end{gathered}
$$

$$
\begin{aligned}
& D=7766 \mathrm{~kg} / \mathrm{m}^{3} \\
& \Delta=1146 \mathrm{~kg} / \mathrm{m}^{3}
\end{aligned}
$$

Acknowledgments. We thank D. Anderson and D. Stevenson for helpful discussions and thank R. Jeanloz, E. Knittle, and Q. Williams for copies of their unpublished manuscripts and an anonymous reviewer for many helpful suggestions. Support for this work was provided by the National Science Foundation grant EAR9204586. Contribution 4803 of the Division of Geological and Planetary Sciences, California Institute of Technology.

\section{References}

Ahrens, T. J., Equations of state of iron sulfide and constraints on the sulfur content of the earth, J. Geophys. Res., 84, 985-998, 1979.

Ahrens, T. J., H. Tan, and J. D. Bass, Analysis of shock temperature data for iron, High Press. Res., 2, 145-157, 1990.

Al'tshuler, L. V., K. K. Krupnikov, B. N. Ledenev, V. I. Zhuchikhin, and M. I. Brozhnik, Dynamic compressibility and equation of state of iron under high pressure, Sov. Phys. JETP, 34(7), 606-619, 1958.

Al'tshuler, L. V., A. A. Bakanova, and R. F. Trunin, Shock adiabats and zero isotherms of seven metals at high pressures, Sov. Phys. JETP, 15, 65-74, 1962.

Al'tshuler, L. V., A. A. Bakanova, I. P. Dudoladov, E. A. Dynin, R. F. Trunin, and B. S. Chekin, Shock adiabatic curves of metals: New data, statistical analysis, and general laws, J. Appl. Mech. Tech. Phys. (Engl. Transl.), 22, 141-169, 1981.

Anderson, D. L., A seismic equation of state, Geophys. J. $R$. Astron. Soc., 13, 9-30, 1967.

Anderson, O. L., Properties of iron at the Earth's core conditions, Geophys. J. R. Astron. Soc., 84, 561-579, 1986.

Basin, A. S., Y. L. Kolotov, and S. V. Stankus, The density and thermal expansion of iron and ferrous alloys, High Temp. High Pressures, 11, 465-470, 1979.

Bass, J. D., B. Svendsen, and T. J. Ahrens, The temperature of shock compressed iron, in High Pressure Research in Mineral Physics, edited by M. H. Manghnani and Y. Syono, pp. 393-402, Terra Scientific, Tokyo, 1987.

Boness, D. A., J. M. Brown, and A. K. McMahan, The electronic thermodynamics of iron under Earth core conditions, Phys. Earth Planet. Inter., 42, 227-240, 1986.

Brown, J. M., and R. G. McQueen, Phase transitions, Grüneisen parameter, and elasticity for shocked iron between $77 \mathrm{GPa}$ and $400 \mathrm{GPa}$, J. Geophys. Res., 91, 7485-7494, 1986.

Casas, J., N. M. Kéita, and S. G. Steinemann, Sound velocities in liquid titanium, vanadium, and chromium, Phys. Chem. Liq., 14, 155-158, 1984.

Desai, P. D., Thermodynamic properties of iron and silicon, $J$. Phys. Chem. Ref. Data, 15, 967-983, 1986.

Drotning, W. D., Thermal expansion of iron, cobalt, nickel, and copper at temperatures to $600 \mathrm{~K}$ above melting, High Temp. High Pressures, 13, 441-458, 1981.

Dziewonski, A. M., and D. L. Anderson, Preliminary reference Earth model, Phys. Earth Planet. Inter., 25, 297-356, 1981.

Falzone, A. J., and F. D. Stacey, Second order elasticity theory: An improved formulation of the Grüneisen parameter at high pressure, Phys. Earth Planet. Inter., 24, 284-290, 1981.

Fillipov, S. J., N. B. Kazakov, and L. A. Pronin, Velocities of ultrasonic waves, compressibility of liquid metals, and their relation to various physical properties (in Russian), Izv. Vyssh. Uchebn. Zaved. Chern. Metall., 9, 8-14, 1966.

Gillan, M. J., A new method of solving the liquid structure integral equations, Mol. Phys., 38, 1781-1794, 1979.

Grover, R., Experimental and theoretical constraints on the iron equation of state, J. Geophys. Res., 95, 21,743-21,748, 1990.

Hafner, J., and V. Heine, Theory of the atomic interactions in (s, p)-bonded metals, J. Phys. F: Met. Phys., 16, 1429-1458, 1986.

Hausleitner, C., and J. Hafner, Soft-sphere reference system in thermodynamic variational calculations, II, Liquid transition metals, J. Phys. F: Met. Phys., 18, 1025-1036, 1988.

Hixson, R. S., M. A. Winkler, and M. L. Hodgdon, Sound speed 
and thermophysical properties of iron and nickel, Phys. Rev. B, 42, 6485-6491, 1990.

Hofmeister, A. M., Pressure derivatives of the bulk modulus, $J$. Geophys. Res., 96, 21,893-21,907, 1991.

Ichimaru, S., and K. Utsumi, Analytic expression for the dielectric screening function of strongly coupled electron liquids at metallic and lower densities, Phys. Rev. B, 24, 7385-7388, 1981.

Ivakhnenko, I. S., and V. I. Kashin, in Zakonomernosti Vzaimodeistviya Zhidkogo Metalla s Gazami i Shlakami, edited by I. S. Kulikov, pp. 135-142, Nauka, Moscow, 1976.

Jank, W., C. Hausleitner, and J. Hafner, Electronic structure of the liquid 3d and 4d transition metals, J. Phys.: Condens. Matter., 3, 4477-4490, 1991.

Jeanloz, R., Properties of iron at high pressures and the state of the core, J. Geophys. Res., 84, 6059-6069, 1979.

Jephcoat, A., and P. Olsen, Is the inner core of the Earth pure iron?, Nature, 325, 332-335, 1987.

Kéita, N. M., H. Morita, and S. G. Steinemann, Cohesive properties of liquid transition metals, paper presented at 4th International Conference on Rapidly Quenched Metals JPN Inst. of Metals, Sendai, 1982.

Krupnikov, K. K., A. A. Bakanova, M. I. Brazhnik, and R. F. Trunin, An investigation of the shock compressibility of titanium, molybdenum, tantalum, and iron, Sov. Phys. Dokl., 8, 205-207, 1963.

Kurz, W., and B. Lux, Die Schallgeswindigkeit von Eisen und Eisenlegierungen in festen und flüssigen Zustand, High Temp. High Pressures, 1, 387-399, 1969.

Lado, F., S. M. Foiles, and N. W. Ashcroft, Solution of the reference-hypernetted chain equation with minimization of free energy, Phys. Rev. A, 28, 2374-2379, 1983.

Lucas, L. D., Densité de métaux à haute température (dans les états solide et liquide), Mem. Sci. Rev. Metall., 69, 479-492, 1972.

Marsh, S. P., LASL Shock Hugoniot Data, 658 pp., University of California Press, Berkeley, 1980.

McQueen, R. G., S. P. Marsh, J. W. Taylor, J. N. Fritz, and W. J. Carter, The equation of state of solids from shock wave studies, in High Velocity Impact Phenomena, edited by R. Kinslow, pp. 293-417, Academic, San Diego, Calif., 1970.

Mulargia, F., Is the common definition of the Mie-Grüneisen equation of state inconsistent?, Geophys. Res. Lett., 4, 590-592, 1977.

Pettifor, D. G., and M. A. Ward, An analytic pair potential for simple metals, Solid State Commun., 49, 291-294, 1984.

Press, W. H., B. P. Flannery, S. A. Teukolsky, and W. T. Vetterling, Numerical Recipes, 818 pp., Cambridge University Press, New York, 1986.

Rosenfeld, Y., and N. W. Ashcroft, Theory of simple classical fluids: Universality in the short-range structure, Phys. Rev. A, 20, 1208-1235, 1979.

Shaner, J. W., R. S. Hixson, M. A. Winkler, D. A. Boness, and J. M. Brown, Birch's law for fluid metals, in Shock Waves in Condensed Matter 1987, edited by S. C. Schmidt and N. C. Holmes, pp. 135-138, Elsevier, New York, 1988.

Stacey, F. D., Interpretation of adiabatic decompression measurements of the Grüneisen parameter, J. Geophys. Res., 86, 1039 1041, 1981.

Stacey, F. D., B. J. Brennan, and R. D. Irvine, Finite strain theories and comparisons with seismological data, Geophys. Surv., 4, 189-232, 1982.

Stevenson, D. J., Applications of liquid state theory to the Earth's core, Phys. Earth Planet. Inter., 22, 42-52, 1980.

Stevenson, D. J., Models of the Earth's core, Science, 214, 611-619, 1981.

Svendsen, B., W. W. Anderson, T. J. Ahrens, and J. D. Bass, Ideal $\mathrm{Fe}-\mathrm{FeS}$ and $\mathrm{Fe}-\mathrm{FeO}$ phase relations and the Earth's core, Phys. Earth Planet. Inter, 55, 154-186, 1989.

Tillotson, J. H., Metallic equations of state for hypervelocity impact, in General Atomic Report, Rep. GA 3216, 139 pp., Gen. Dyn. Corp., San Diego, Calif., 1962.

Tsu, Y., K. Takano, and Y. Shiraishi, The velocities of ultrasound in molten iron, cobalt, and nickel (in Japanese), Bull. Res. Inst. Miner. Dressing Metall. Tohoku Univ., 41, 1-8, 1985.

Waseda, Y., and K. Suzuki, Atomic distribution and magnetic moment in liquid iron by neutron diffraction, Phys. Status Solidi., 39, 669-675, 1970.

Weir, G. F., M. A. Howson, B. L. Gallagher, and G. J. Morgan, Hybridization in amorphous metals, Philos. Mag. B, 47, 163-176, 1983.

Williams, Q., R. Jeanloz, J. Bass, B. Svendsen, and T. J. Ahrens, The melting curve of iron to 250 gigapascals: A constraint on the temperature at Earth's center, Science, 236, 181-182, 1987.

Yoo, C. S., N. C. Holmes, M. Ross, D. J. Webb, and C. Pike, Shock temperatures and melting of iron at Earth core conditions, Phys. Rev. Lett., 70, 3931-3934, 1993.

Zerah, G., and J. P. Hansen, Self-consistent integral equations for fluid pair distribution functions: Another attempt, J. Chem. Phys., 84, 2336-2343, 1986.

T. J. Ahrens and W. W. Anderson, Lindhurst Laboratory of Experimental Geophysics, Seismological Laboratory, California Institute of Technology, Pasadena, CA 91125. (tel. 818-395-2986, fax 818-564-0715)

(Received October 5, 1992; revised September 20, 1993; accepted November 5, 1993.) 